EPSC Abstracts

Vol. 14, EPSC2020-419, 2020

https://doi.org/10.5194/epsc2020-419

Europlanet Science Congress 2020

(C) Author(s) 2020. This work is distributed under

the Creative Commons Attribution 4.0 License.

\title{
Narrow-band photometry of Long Period Comets with TRAPPIST telescopes in 2019-2020
}

\author{
Youssef Moulane ${ }^{1,2,3}$, Emmanuel Jehin ${ }^{2}$, Francisco José Pozuelos ${ }^{2}$, Jean Manfroid ${ }^{2}$, Zouhair \\ Benkhaldoun ${ }^{3}$, and Bin Yang ${ }^{1}$ \\ ${ }^{1}$ European Southern Observatory (ESO), Chile (ymoulane@eso.org) \\ ${ }^{2}$ Space sciences, Technologies \& Astrophysics Research (STAR) Institute, University of Liège, Belgium \\ ${ }^{3}$ Oukaimeden Observatory, High Energy Physics \& Astrophysics Laboratory, Cadi Ayyad University, Morocco
}

Long Period Comets (LPCs) have orbital periods longer than 200 years, perturbed from their resting place in the Oort cloud. Such gravitational influences may send these icy bodies on a path towards the center of the Solar system in highly elliptical orbits. In this work, we present the activity and composition evolution of several LPCs observed with both TRAPPIST telescopes (TS and TN) during the period of 2019-2020. These comets include: C/2017 T2 (PANSTARRS), C/2018 Y1 (Iwamoto), $\mathrm{C} / 2018$ W2 (Africano), and disintegrated comet C/2019 Y4 (ATLAS). We monitored the OH, NH, CN, $\mathrm{C}_{2}$ and $\mathrm{C}_{3}$ production rates evolution and their chemical mixing ratios with respect to their distances to the Sun as well as the dust production rate proxy $(A(0) f p)$ during the journey of these comets into the inner Solar system.

C/2017 T2 (PANSTARRS) is a very bright comet which was discovered on October 2, 2017 when it was 9.20 au from the Sun. We started observing this comet with TS at the beginning of August 2019 when it was at 3.70 au. The comet made the closest approach to the Earth on December 28, 2019 at a distance of 1.52 au and it passed the perihelion on May 4, 2020 at 1.61 au. The water production rate of the comet reached a maximum of $(4,27 \pm 0,12) 10^{28}$ molecules/s and its dust production rate $(A(0) f p(R C))$ also reached the peak of $5110 \pm 25 \mathrm{~cm}$ on January 26,2020 , when the comet was at 2.08 au from the Sun (-100 days pre-perihelion). At the time of writing, we still monitoring the activity of the comet with TN at heliocentric distance of 1.70 au. Our observations show that C/2017 T2 is a normal LPC.

C/2018 Y1 (Iwamoto) is a nearly parabolic comet with a retrograde orbit discovered on December 18, 2018 by Japanese amateur astronomer Masayuki Iwamoto. We monitored the activity and composition of Iwamoto with both TN and TS telescopes from January to March 2019. The comet reached its maximum activity on January 29,2019 when it was at 1.29 au from the Sun (-8 days pre-perihelion) with $\mathrm{Q}\left(\mathrm{H}_{2} \mathrm{O}\right)=(1,68 \pm 0,05) 10^{28}$ molecules $/ \mathrm{s}$ and $\mathrm{A}(0) \mathrm{fp}(\mathrm{RC})=92 \pm 5 \mathrm{~cm}$. These measurements show that it was a dust-poor comet compared to the typical LPCs.

C/2018 W2 (Africano) was discovered on November 27, 2018 at Mount Lemmon Survey with a visual magnitude of 20. The comet reached its perihelion on September 6, 2019 when it was at 1.45 au from the Sun. We monitored the comet from July $2019\left(r_{h}=1.71 \mathrm{au}\right)$ to January $2020\left(r_{h}=2.18\right.$ $\mathrm{au}$ ) with both TN and TS telescopes. The comet reached its maximum activity on September 21,15 days post-perihelion $\left(r_{h}=1.47 \mathrm{au}\right)$ with $\mathrm{Q}\left(\mathrm{H}_{2} \mathrm{O}\right)=(0,40 \pm 0,03) 10^{28}$ molecules $/ \mathrm{s}$. 
C/2019 Y4 (ATLAS) is a comet with a nearly parabolic orbit discovered on December 18, 2019 by the ATLAS survey. We started to follow its activity and composition with broad-and narrow-band filters with the TN telescope on February 22, 2019 when it was at 1.32 au from the Sun until May 3, 2020 when the comet was at a heliocentric distance of 0.90 au inbound. The comet activity reached a maximum on March $22\left(r_{h}=1.65\right.$ au $) 70$ days before perihelion. At that time, the water-production rate reached $(1,53 \pm 0,04) 10^{28}$ molecules/s and the $A(0) \mathrm{fp}$ reached $(1096 \pm 14) \mathrm{cm}$ in the red filter. After that, the comet began to fade and disintegrated into several fragments. 